\title{
Ultrasound imaging of the carpal tunnel during median nerve compression
}

\author{
Benjamin M. Sucher
}

Published online: 1 December 2009

(C) The Author(s) 2009. This article is published with open access at Springerlink.com

\begin{abstract}
Median nerve (MN) compression is a recognized component of carpal tunnel syndrome (CTS). In order to document compressive changes in the MN during hand activity, the carpal tunnel was imaged with neuromuscular ultrasound (NMUS). Ten patients with CTS and five normal controls underwent NMUS of the MN at rest and during dynamic stress testing (DST). DST maneuvers involve sustained isometric flexion of the distal phalanges of the first three digits. During DST in the CTS patients, NMUS demonstrated MN compression between the contracting thenar muscles ventrally and the taut flexor tendons dorsally. The mean MN diameter decreased nearly $40 \%$, with focal narrowing in the mid-distal carpal canal. Normal controls demonstrated no MN compression and a tendency towards $\mathrm{MN}$ enlargement, with an average diameter increase of $17 \%$. Observing the pathologic mechanism of MN injury during common prehensile hand movements could help better understand how to treat and prevent CTS.
\end{abstract}

Keywords Carpal tunnel - Median nerve .

Electrodiagnosis · Ultrasound

\section{Introduction}

Carpal tunnel syndrome (CTS) is caused by median nerve (MN) compression at the wrist [1, p. 67] The carpal tunnel (CT) is a narrow unyielding space that entraps the MN

B. M. Sucher $(\square)$

EMG Labs of AARA, 10599 N. Tatum Blvd., Suite F-150,

Paradise Valley, AZ 85253, USA

e-mail: drsucher@msn.com between the transverse carpal ligament (TCL) ventrally and carpal bones dorsally. The MN typically incurs damage more readily than the less fragile flexor tendons. Vigorous or repetitive finger activity can also contribute to CTS, because pressure from the flexor tendons often irritates the adjacent $\mathrm{MN}$, and inflammatory swelling of the tendon sheaths increases compartment pressure within the CT $[1,2]$. Previous studies have documented that intracarpal pressures are elevated in patients with CTS as compared to non-CTS subjects [1, 2]. However, studies that image the MN during activity to identify mechanical compression are lacking. High resolution neuromuscular ultrasound (NMUS) imaging is available to directly observe responses to hand maneuvers that challenge the MN. The cases in this report demonstrate how the $\mathrm{MN}$ responds to prehensile hand activity in CTS and non-CTS individuals, thereby providing a better understanding of the pathomechanics that lead to nerve compression.

\section{Methods}

Ten patients with CTS and five patients without CTS were examined by history and physical as well as electrodiagnostic (EDX) testing, to confirm the presence or absence of CTS. Skin temperature was maintained at $32.0^{\circ} \mathrm{C}$ or above during EDX studies. Median motor distances were $8 \mathrm{~cm}$ across the wrist to the abductor pollicis brevis and ulnar motor distances were $8 \mathrm{~cm}$ across the wrist to the abductor digiti minimi, sensory distances to D-1 (thumb) were $10 \mathrm{~cm}$, and sensory distances were $14 \mathrm{~cm}$ to D-2 (index finger), D-4 (ring finger), and D-5 (little finger). All ulnar sensory latencies were obtained from D-5 unless otherwise specified at D-4. In some cases, a mixed nerve study (primarily sensory) was performed, which is a comparison of median to 
ulnar conduction across a short $(8 \mathrm{~cm})$ segment at the wrist. Needle electromyographic exam of the abductor pollicis brevis muscle was not performed unless the median distal motor latency (DML) was $>5.0 \mathrm{~ms}$ or there was greater than mild conduction block (over 35\%), or if there was at least a moderate decrease $(>35 \%)$ in the median sensory response amplitude. Diagnosis of CTS was confirmed if the median DML was $>4.3 \mathrm{~ms}$ or $>0.9 \mathrm{~ms}$ above the ulnar DML, or the median distal sensory latency (DSL) to D-1 was $>2.9$ ms or $>0.4 \mathrm{~ms}$ above the radial D-1 DSL, or the median D-2 DSL was $>3.7 \mathrm{~ms}$ or $>0.4 \mathrm{~ms}$ above the ulnar D-5 DSL. In some cases, supplemental studies included the median-ulnar DSL to D-4 (normal difference $<0.5 \mathrm{~ms}$ ) and the median-ulnar mixed nerve latencies (normal difference $<0.4 \mathrm{~ms}$ ), in order to calculate the combined sensory index (CSI), which is considered abnormal at $>1.0 \mathrm{~ms}$ [3].

Immediately following EDX, the MN was imaged with high resolution neuromuscular ultrasound (NMUS), using a Sonosite M-Turbo, 6-13 MHz system, measurement accuracy $\pm 2 \%$. Initially, transverse images were obtained to measure the $\mathrm{MN}$ cross-sectional area (CSA) in the proximal $\mathrm{CT}$ at the level of the pisiform. The normal CSA upper limit is $11 \mathrm{~mm}^{2}[4,5]$. If a patient had a borderline (high normal) CSA of $10-11 \mathrm{~mm}^{2}$, then the MN was usually imaged in the distal forearm to determine the wrist-forearm ratio (WFR). The WFR is the median nerve diameter at the wrist divided by the median nerve diameter in the distal forearm [6]. The normal WFR should be $<1.5$ [6]. The MN was imaged in the carpal tunnel in a neutral relaxed position, and repeated during dynamic stress testing (DST), usually in the longitudinal plane, as stress images in the transverse plane were less consistently obtained. DST involves a sustained isometric contraction of the thumb flexors and abductors as well as the long flexors to the 2 nd and 3rd digits, utilizing a hard rubber ball for resistance. The relatively unyielding ball maintains separation of the thumb from the fingertips, which prevents the thenar mass from moving medially and pushing the ultrasound transducer off the nerve. Linear measurements are obtained before and during DST of the MN diameter at the widest point in the mid-distal carpal canal, by aligning the markers at the inner edges of the hyperechoic rim (epineurium) of the MN.

\section{Results}

Normal subjects

NMUS images on the five normal wrists are illustrated in Figs. 1, 2, 3, 4, and 5 and $\mathrm{MN}$ diameter values are listed in Table 1. These patients presented with upper limb complaints that were not consistent with CTS. They completed EDX studies that revealed normal median nerve function (no abnormalities). NMUS studies were performed to confirm that the MN appeared normal (no compression).

Subject \#1 (Fig. 1), MN diameter at rest (pre-stress) was $0.23 \mathrm{~cm}$, and during stress it was $0.31 \mathrm{~cm}$, for a $34.8 \%$ increase. Subject \#2 (Fig. 2), MN diameter was $0.13 \mathrm{~cm}$ at rest, and $0.17 \mathrm{~cm}$ during stress, for a $30.8 \%$ increase. Subject \#3 (Fig. 3), MN diameter was $0.13 \mathrm{~cm}$ at rest and during stress stayed at $0.13 \mathrm{~cm}$, for a $0 \%$ change. Subject \#4 (Fig. 4), MN diameter was $0.15 \mathrm{~cm}$ at rest and $0.17 \mathrm{~cm}$ during stress, for a $13.3 \%$ increase. Subject \#5 (Fig. 5), $\mathrm{MN}$ diameter was $0.16 \mathrm{~cm}$ at rest, and $0.17 \mathrm{~cm}$ during stress, for a $6.25 \%$ increase.

The mean change during stress for these normal subjects was $17.03 \%$ increase in MN diameter (see Table 1).

(a) Normal Subject \#1 Pre-Stress Image:

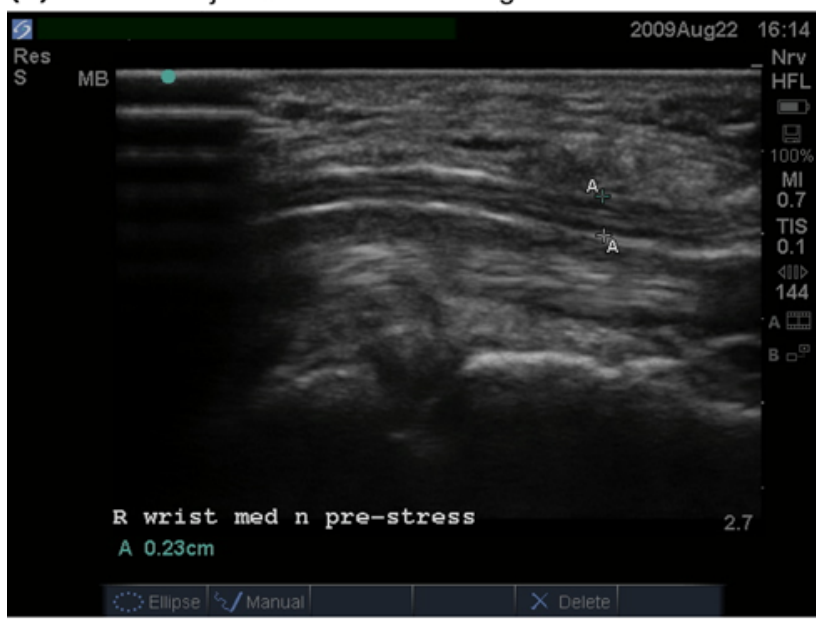

(b) Normal Subject \#1 Stress Image:

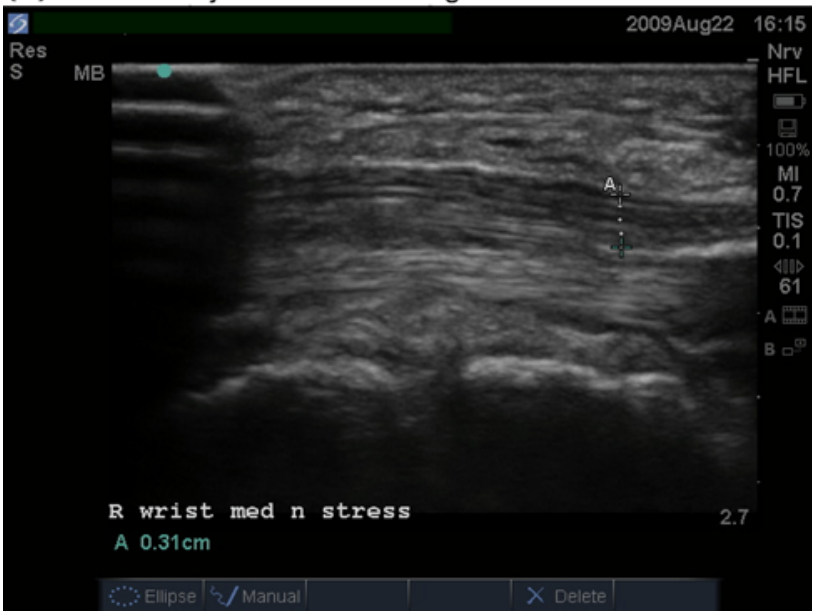

Fig. 1 Normal subject \#1. Longitudinal view of the median nerve pre-stress (a) and during stress (b). The median nerve is the darker, hypoechoic linear (or tubular) structure bordered by a thin hyperechoic rim (epineurium) traversing from the left to the right side of the image. The brighter, hyperechoic tubular structure with a fibrillar pattern just below the nerve is the flexor tendon. The nerve diameter increased from $0.23 \mathrm{~cm}$ pre-stress to $0.31 \mathrm{~cm}$ during stress 


\section{(a) Normal Subject \#2 Pre-Stress image:}

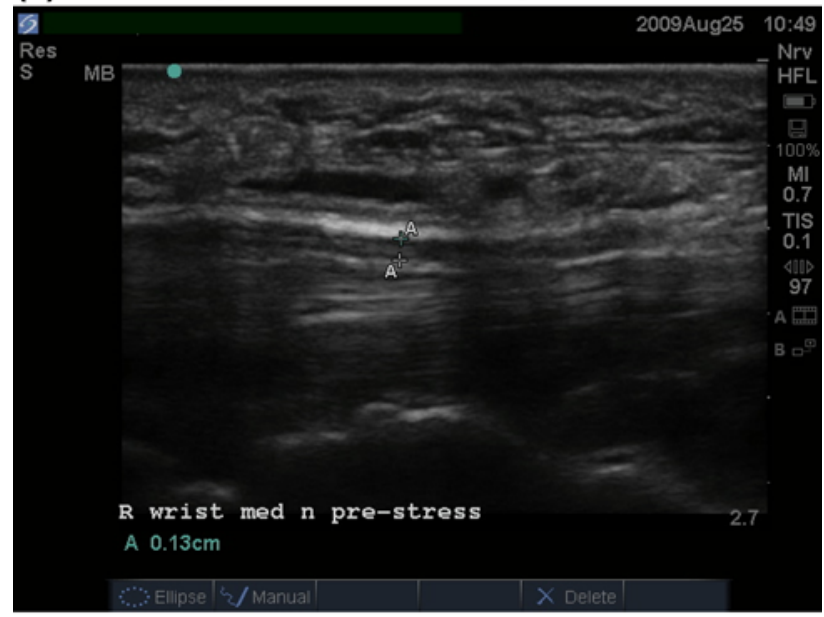

(b)Normal Subject \#2 Stress Image:

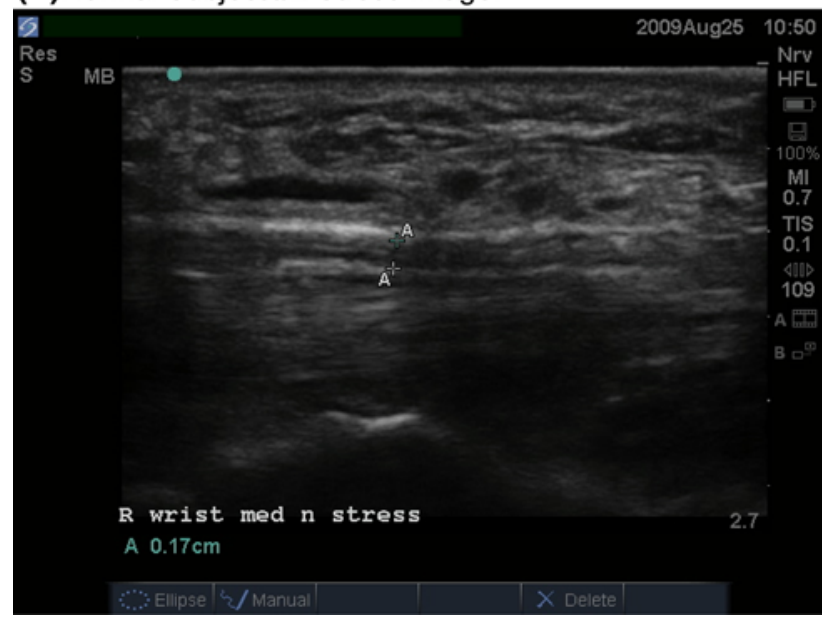

Fig. 2 Normal subject \#2. Longitudinal view of the median nerve pre-stress (a) and during stress (b). The median nerve is the darker, hypoechoic linear (or tubular) structure bordered by a thin hyperechoic rim (epineurium) traversing from the left to the right side of the image. The brighter, hyperechoic tubular structure with a fibrillar pattern just below the nerve is the flexor tendon. The nerve diameter increased from $0.13 \mathrm{~cm}$ pre-stress to $0.17 \mathrm{~cm}$ during stress

\section{CTS patients}

NMUS images on the 10 CTS patients are illustrated in Figs. 6, 7, 8, 9, 10, 11, 12, 13, 14, and 15 and MN diameter values are listed in Table 2. All patients had symptoms and EDX findings consistent with CTS, and the MN narrowed during the DST portion of the NMUS exam. The mean decrease in MN diameter during DST was 39.5\% (see Table 2).

Case \#1 is a 67-year-old female with left upper extremity pain, numbness, and tingling for several months, who presented with a positive Phalen test and positive Tinel test at the carpal canal. Electrodiagnostic studies (EDX) confirmed median neuropathy at the left wrist: the median distal motor latency (DML) was $3.8 \mathrm{~ms}$ (ulnar (a) Normal Subject \#3 Pre-Stress Image:

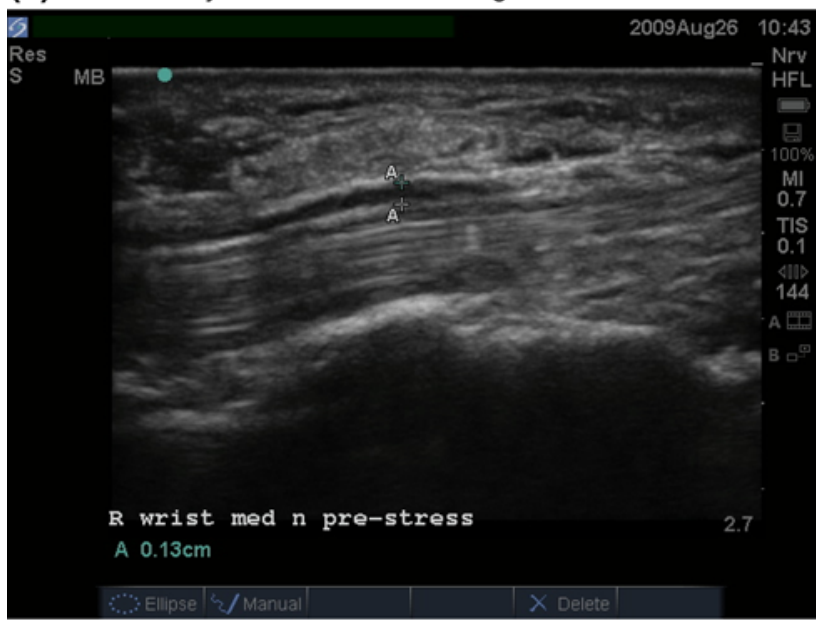

\section{(b) Normal Subject \#3 Stress Image:}

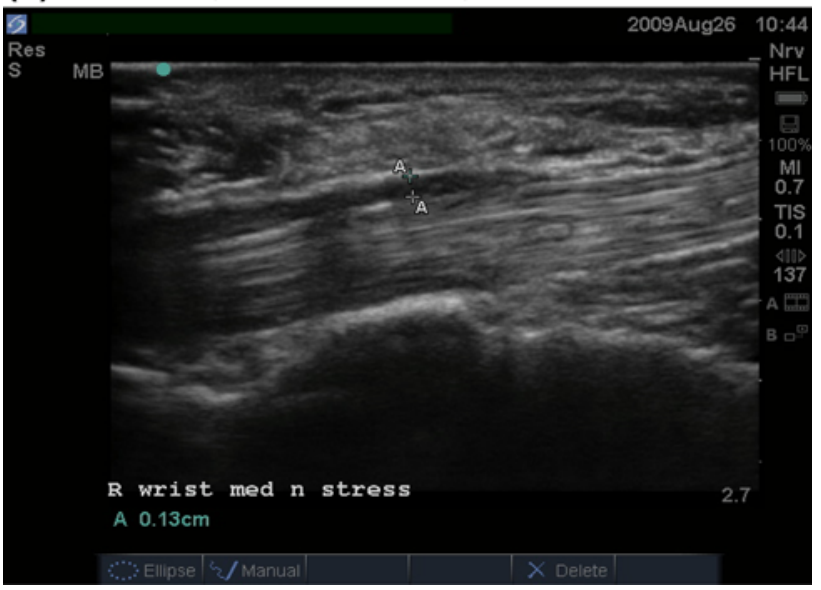

Fig. 3 Normal Subject \#3. Longitudinal view of the median nerve pre-stress (a) and during stress (b). The median nerve is the darker, hypoechoic linear (or tubular) structure bordered by a thin hyperechoic rim (epineurium) traversing from the left to the right side of the image. The brighter, hyperechoic tubular structure with a fibrillar pattern just below the nerve is the flexor tendon. The nerve diameter remained unchanged at $0.13 \mathrm{~cm}$ from the neutral (pre-stress) image to the stress image

DML $2.8 \mathrm{~ms}$ ) and the median distal sensory latency (DSL) to the thumb (D-1) was $3.2 \mathrm{~ms}$ (radial $2.7 \mathrm{~ms}$ ). NMUS of the left wrist revealed borderline (high normal) cross-sectional area (CSA) of the MN $\left(11 \mathrm{~mm}^{2}\right)$ at the level of the pisiform, and an elevated wrist-forearm ratio at 2.75. These findings were compatible with a diagnosis of very mild CTS.

Longitudinal imaging of the $\mathrm{MN}$ (Fig. 6) reveals a prestress nerve diameter of $0.16 \mathrm{~cm}$ that decreased to $0.11 \mathrm{~cm}$ during stress, which represents a $31.25 \%$ compressive narrowing.

Case \#2 is a 68-year-old female with numbness, tingling, and weakness in the right hand for 4 years. Phalen and Tinel testing was normal. Median DML was $3.9 \mathrm{~ms}$ 
(a) Normal Subject \#4 Pre-Stress Image:

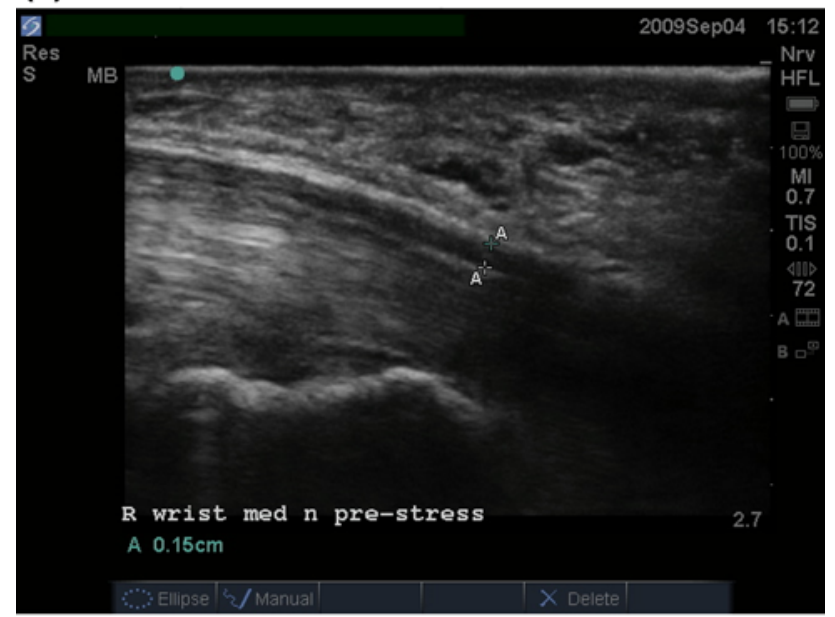

(b) Normal Subject \#4 Stress Image:

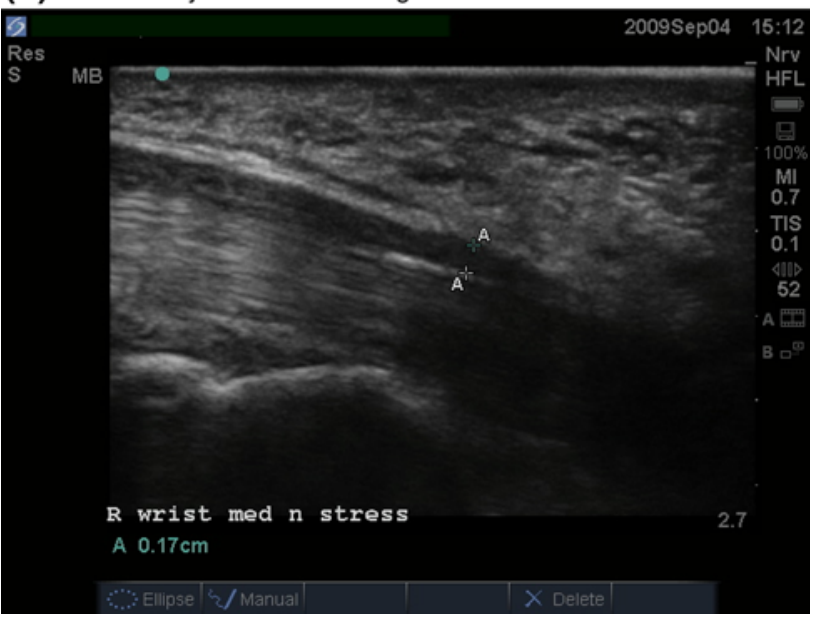

Fig. 4 Normal subject \#4. Longitudinal view of the median nerve pre-stress (a) and during stress (b). The median nerve is the darker, hypoechoic linear (or tubular) structure bordered by a thin hyperechoic rim (epineurium) traversing from the left to the right side of the image. The brighter, hyperechoic tubular structure with a fibrillar pattern just below the nerve is the flexor tendon. The nerve diameter increased from $0.15 \mathrm{~cm}$ pre-stress to $0.17 \mathrm{~cm}$ during stress (a) Normal Subject \#5 Pre-Stress Image:

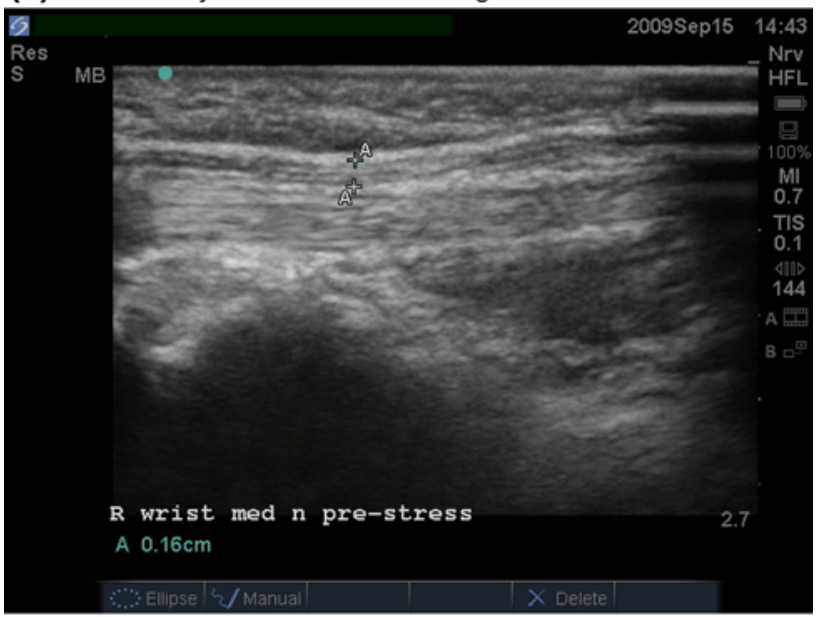

(b) Normal Subject \#5 Stress Image:

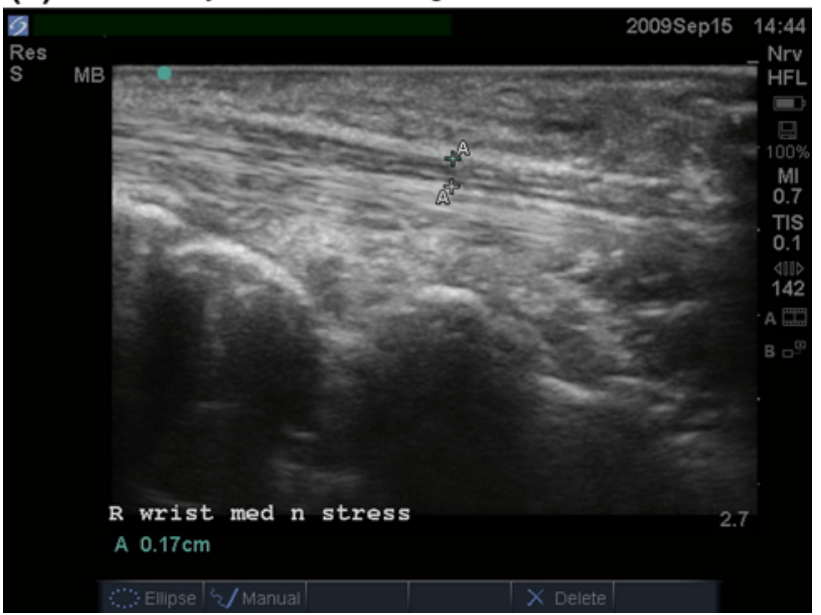

Fig. 5 Normal subject \#5. Longitudinal view of the median nerve pre-stress (a) and during stress (b). The median nerve is the darker, hypoechoic linear (or tubular) structure bordered by a thin hyperechoic rim (epineurium) traversing from the left to the right side of the image. The brighter, hyperechoic tubular structure with a fibrillar pattern just below the nerve is the flexor tendon. The nerve diameter increased from $0.16 \mathrm{~cm}$ pre-stress to $0.17 \mathrm{~cm}$ during stress

Table 1 Normal subjects' measurement data

\begin{tabular}{lllllllll}
\hline Subject\# & H\&P & NCS & EMG & CSA $\left(\mathrm{mm}^{2}\right)$ & WFR & Pre-stress diameter $(\mathrm{cm})$ & Stress diameter $(\mathrm{cm})$ & \% Change \\
\hline 1 & - & - & - & 9 & n/a & 0.23 & 0.31 & 0.17 \\
2 & - & - & - & 11 & 1.2 & 0.13 & 0.13 & +34.8 \\
3 & - & - & - & 9 & 1.1 & 0.13 & 0.17 & 0 \\
4 & - & - & - & 9 & n/a & 0.15 & 0.17 & +13.3 \\
5 & - & - & - & 10 & 1.25 & 0.16 & & +6.25 \\
Mean change & & & & & & & & +17.03 \\
\hline
\end{tabular}

$H \& P$ history and physical, NCS nerve conduction study, EMG needle electromyogram, CSA median nerve cross-sectional area, WFR ratio of median nerve CSA at wrist divided by CSA in distal forearm (normal < 1.5), "_" signifies negative or normal, n/a not applicable or not obtained 


\section{(a) CTS Patient \#1 Pre-Stress Image:}

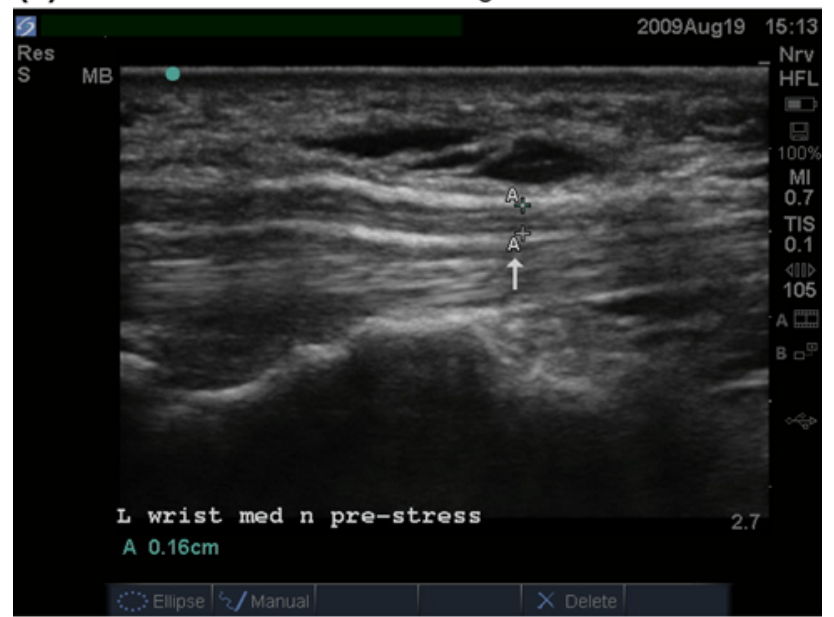

(b)CTS Patient \#1 Stress image:

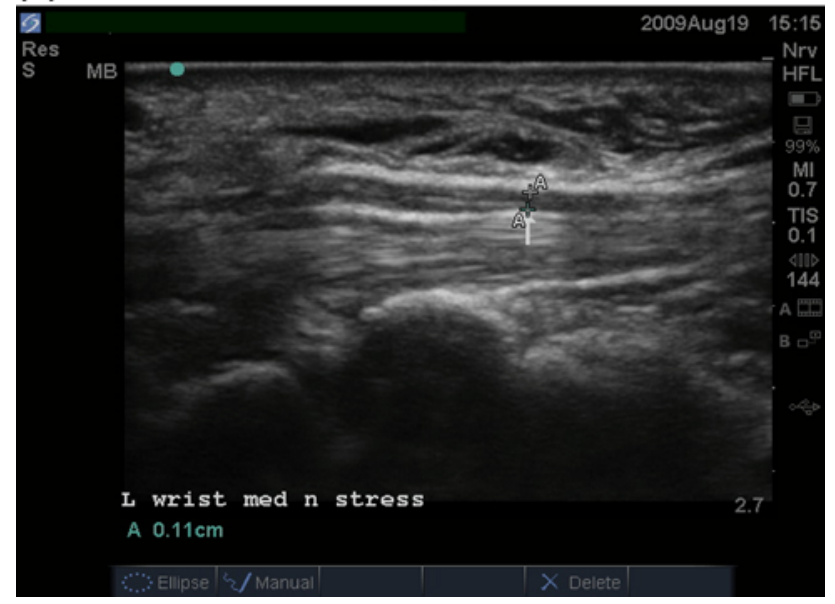

Fig. 6 CTS patient \#1. Longitudinal view of the median nerve prestress (a) and during stress (b). The median nerve is the darker, hypoechoic linear (or tubular) structure bordered by a thin hyperechoic rim (epineurium) traversing from the left to the right side of the image. The brighter, hyperechoic tubular structure with a fibrillar pattern just below the nerve is the flexor tendon. Note that during stress (b) there is compressive narrowing, seen as a "notching" from below (dorsally) or indentation of the median nerve in the segment above the white arrow and to the right, measured as a diameter decrease to $0.11 \mathrm{~cm}$, from a pre-stress diameter of $0.16 \mathrm{~cm}$. It appears that a portion of the flexor tendon has bulged upward (ventrally) into the nerve

(ulnar $3.4 \mathrm{~ms}$ ) and the median D-2 DSL $4.2 \mathrm{~ms}$ (ulnar $3.6 \mathrm{~ms}$ ). NMUS of the right wrist revealed borderline (high normal) CSA of the MN $\left(11 \mathrm{~mm}^{2}\right)$. These findings were consistent with a diagnosis of mild CTS.

Longitudinal imaging of the MN (Fig. 7) reveals a prestress nerve diameter of $0.20 \mathrm{~cm}$ that decreased to $0.12 \mathrm{~cm}$ during stress, which represents a $40 \%$ compressive narrowing.

Case \#3 is a 71-year-old male with pain, numbness, tingling, and weakness in the left upper extremity for several months, who presented with a positive Phalen test (a) CTS Patient \#2 Pre-Stress Image:

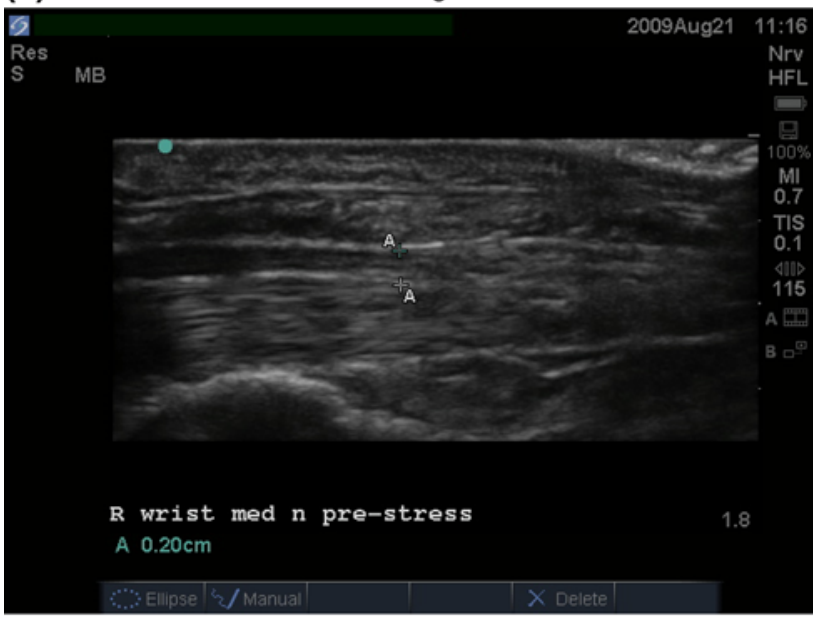

(b) CTS Patient \#2 Stress Image:

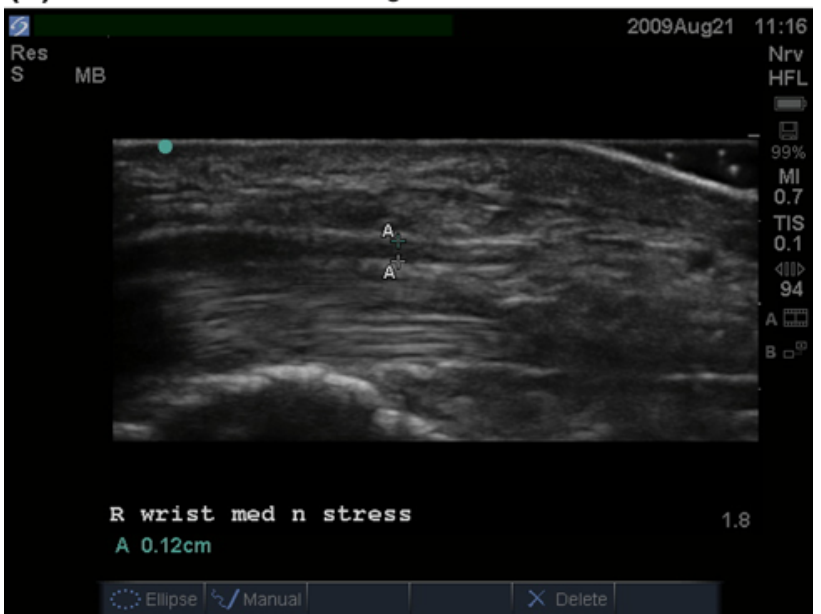

Fig. 7 CTS patient \#2. Longitudinal view of the median nerve prestress (a) and during stress (b). The median nerve is the darker, hypoechoic linear (or tubular) structure bordered by a thin hyperechoic rim (epineurium) traversing from the left to the right side of the image. The brighter, hyperechoic tubular structure with a fibrillar pattern just below the nerve is the flexor tendon. Note that during stress (b) there is compressive narrowing, seen near the center of the image, as the median nerve decreases in diameter to $0.12 \mathrm{~cm}$, from $0.20 \mathrm{~cm}$ pre-stress (a)

(negative Tinel). The median DML was $3.8 \mathrm{~ms}$ (ulnar $2.6 \mathrm{~ms}$ ) and the median D-1 DSL was $3.0 \mathrm{~ms}$ (radial $2.5 \mathrm{~ms}$ ). NMUS of the left wrist revealed borderline (high normal) CSA of the MN $\left(10 \mathrm{~mm}^{2}\right)$ and the WFR was elevated at 2.5 . These findings were consistent with very mild CTS.

Longitudinal imaging of the MN (Fig. 8) reveals a prestress nerve diameter of $0.20 \mathrm{~cm}$ that decreased to $0.13 \mathrm{~cm}$ during stress, which represents a $35 \%$ compressive narrowing.

Case \#4 is a 50-year-old female with left upper extremity pain, numbness, tingling, and weakness for 


\section{(a) CTS Patient \#3 Pre-Stress Image:}

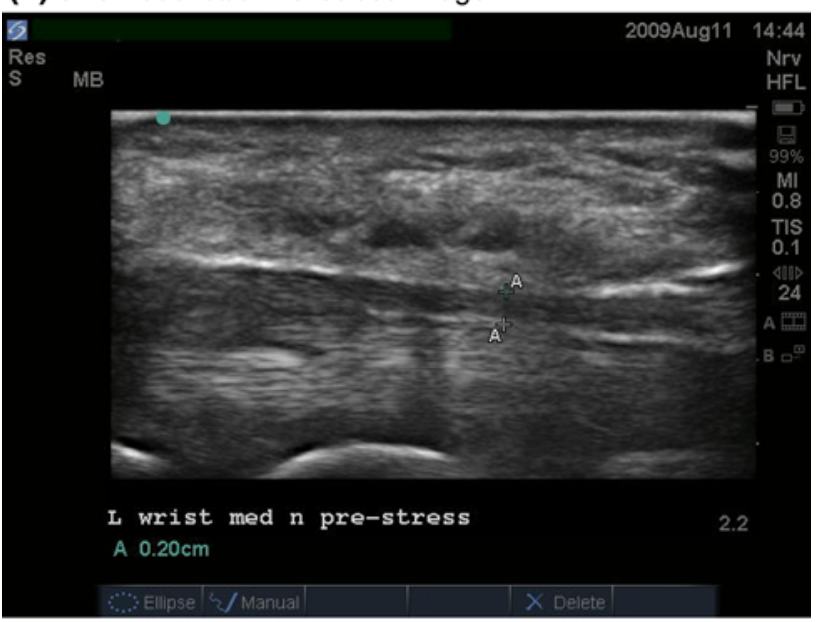

(b) CTS Patient \#3 Stress Image:

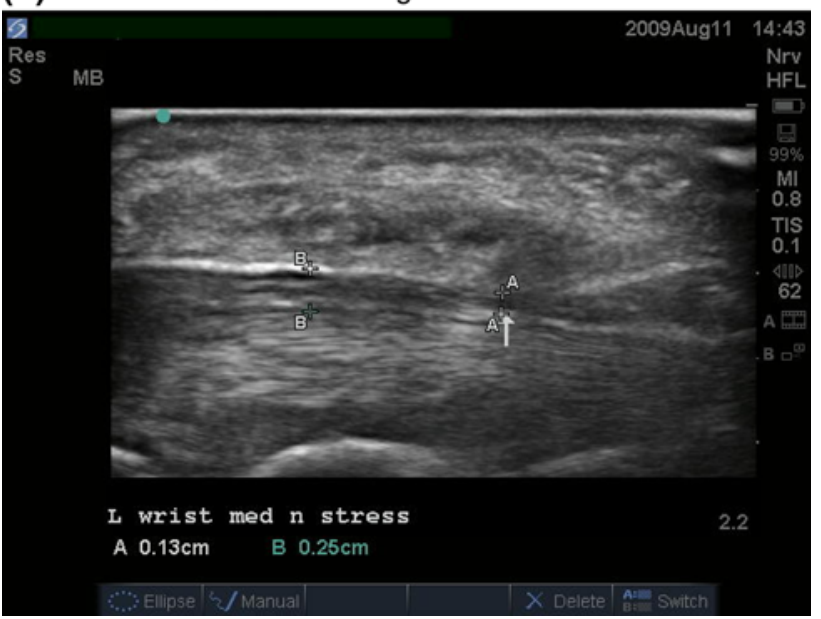

Fig. 8 CTS patient \#3. Longitudinal view of the median nerve prestress (a) and during stress (b). The median nerve is the darker, hypoechoic linear (or tubular) structure bordered by a thin hyperechoic rim (epineurium) traversing from the left to the right side of the image. The brighter, hyperechoic tubular structure with a fibrillar pattern just below the nerve is the flexor tendon. Note that during stress (b) there is pronounced compressive narrowing, seen as a depression or notching along the median nerve at the "A" markers to the right, measured as a diameter decrease to $0.13 \mathrm{~cm}$, from a prestress diameter of $0.20 \mathrm{~cm}$. It appears that during contraction the thenar muscle mass (relatively hyperechoic, large area), located ventrally (above the nerve in this image), is bulging dorsally into the tunnel and against the median nerve

5 months, who presented with a positive Phalen test (negative Tinel). Median DML was $3.9 \mathrm{~ms}$ (ulnar $2.8 \mathrm{~ms}$ ), median D-1 DSL was $3.3 \mathrm{~ms}$ (radial $2.7 \mathrm{~ms}$ ). NMUS of the left wrist revealed borderline CSA of the MN $\left(10 \mathrm{~mm}^{2}\right)$ and the WFR was elevated at 2.0. This data was consistent with mild CTS.

Longitudinal imaging of the MN (Fig. 9) reveals a prestress nerve diameter of $0.20 \mathrm{~cm}$ that decreased to $0.13 \mathrm{~cm}$ (a) CTS Patient \#4 Pre-Stress Image:

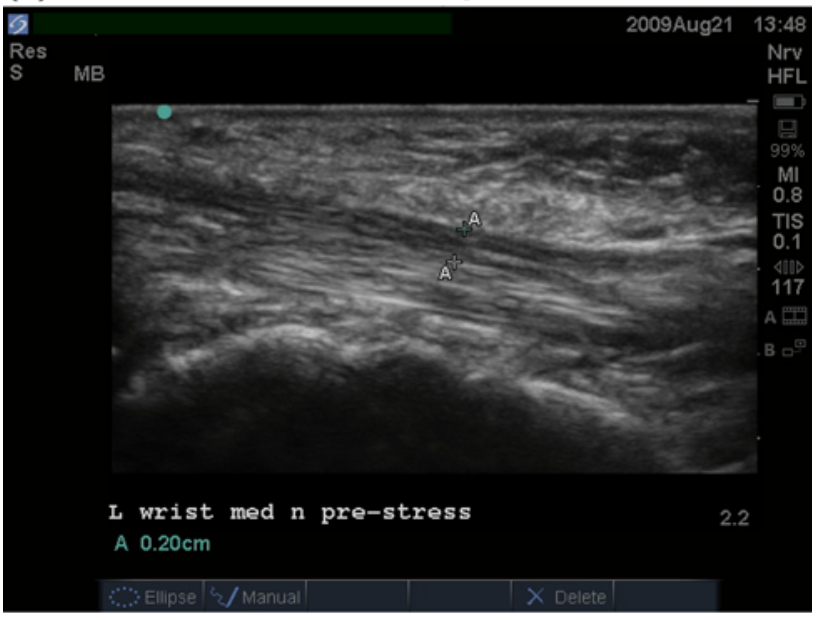

(b) CTS Patient \#4 Stress Image:

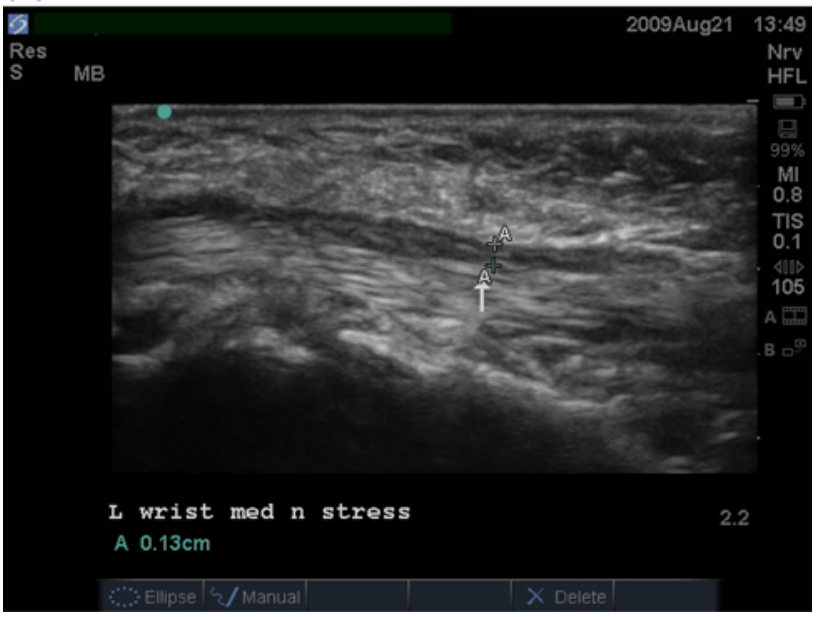

Fig. 9 CTS patient \#4. Longitudinal view of the median nerve prestress (a) and during stress (b). The median nerve is the darker, hypoechoic linear (or tubular) structure bordered by a thin hyperechoic rim (epineurium) traversing from the left to the right side of the image. The brighter, hyperechoic tubular structure with a fibrillar pattern just below the nerve is the flexor tendon. Note that during stress (b) there is compressive narrowing, seen as a depression or notching of the median nerve at the "A" markers (centrally and slightly to the right), measured as a diameter decrease to $0.13 \mathrm{~cm}$, from a pre-stress diameter of $0.20 \mathrm{~cm}$. It appears that during contraction the thenar muscle mass located ventrally (above the nerve in this image) is bulging dorsally into the tunnel against the median nerve, and a portion of the flexor tendon has tightened and is bulging upward (ventrally) into the nerve, effectively "sandwiching" the nerve between the muscle and tendon

during stress, which represents a 35\% compressive narrowing.

Case \#5 is a 48-year-old female with numbness, tingling, and weakness in the right upper limb for a month. Phalen and Tinel testing was normal. The median DML was $4.0 \mathrm{~ms}$ (ulnar $3.5 \mathrm{~ms}$ ) and median D-1 DSL was $3.3 \mathrm{~ms}$ (radial $2.6 \mathrm{~ms}$ ). NMUS of the right wrist revealed 


\section{(a) CTS Patient \#5 Pre-Stress Image:}

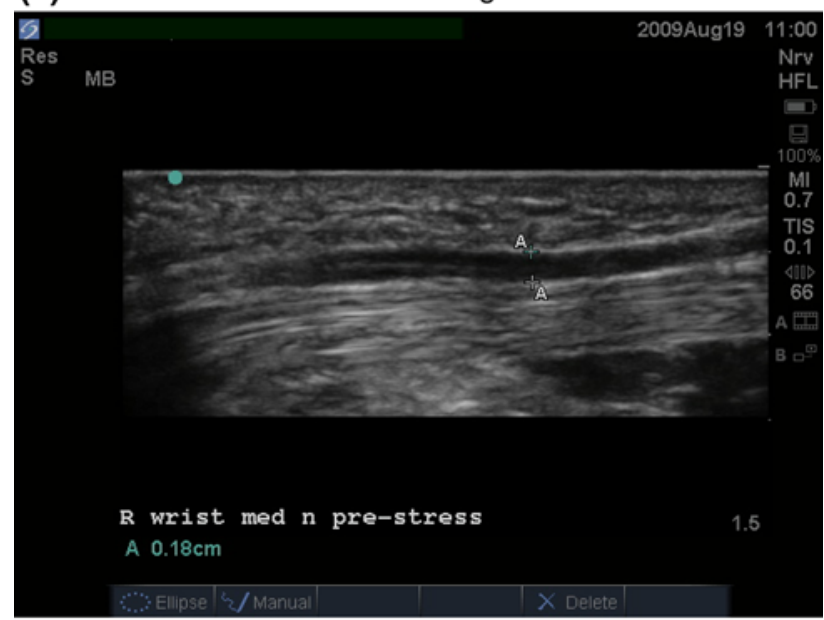

(b) CTS Patient \#5 Stress Image:

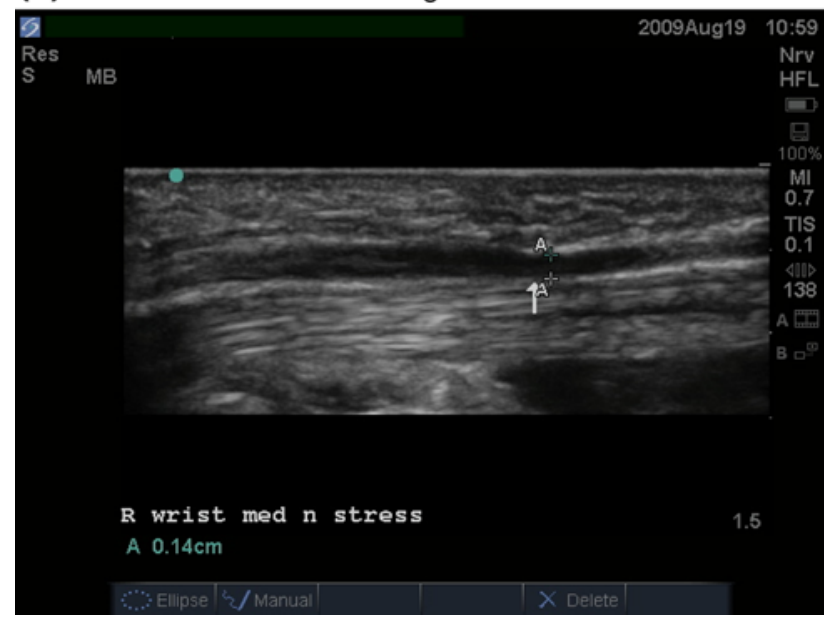

Fig. 10 CTS patient \#5. Longitudinal view of the median nerve prestress (a) and during stress (b). The median nerve is the darker, hypoechoic linear (or tubular) structure bordered by a thin hyperechoic rim (epineurium) traversing from the left to the right side of the image. The brighter, hyperechoic tubular structure with a fibrillar pattern just below the nerve is the flexor tendon. Note that during stress (b) there is compressive narrowing, seen as a depression or notching of the median nerve at the " $\mathrm{A}$ " markers to the right, measured as a diameter decrease to $0.14 \mathrm{~cm}$, from a pre-stress diameter of $0.18 \mathrm{~cm}$. It appears that during contraction the thenar muscle mass located ventrally (above the nerve in this image) is bulging dorsally into the tunnel and against the median nerve

increased CSA of the MN $\left(13 \mathrm{~mm}^{2}\right)$. These findings were consistent with mild CTS.

Longitudinal imaging of the MN (Fig. 10) reveals a prestress nerve diameter of $0.18 \mathrm{~cm}$ that decreased to $0.14 \mathrm{~cm}$ during stress, which represents a $22.2 \%$ compressive narrowing.

Case \#6 is a 57-year-old male, right hand dominant, with bilateral upper extremity pain, numbness, tingling, and weakness for 4 months. Phalen and Tinel testing was normal. The left median DML was $4.9 \mathrm{~ms}$ (ulnar $2.8 \mathrm{~ms}$ ) and the right median was DML $4.9 \mathrm{~ms}$ (ulnar $2.9 \mathrm{~ms}$ ), and the median D-1 DSL was $4.7 \mathrm{~ms}$ left and $4.2 \mathrm{~ms}$ right (radial $2.8 \mathrm{~ms}$ bilaterally). NMUS of the wrists revealed increased CSA of the MNs (13 $\mathrm{mm}^{2}$ each). This data was consistent with mild-moderate CTS, left greater than right.

Longitudinal imaging of the MNs (Fig. 11) reveals a pre-stress nerve diameter on the left of $0.11 \mathrm{~cm}$ that decreased to $0.05 \mathrm{~cm}$ during stress, which represents a $54.5 \%$ compressive narrowing; and pre-stress nerve diameter on the right of $0.09 \mathrm{~cm}$ that decreased to $0.05 \mathrm{~cm}$ during stress, which represents a $44.4 \%$ compressive narrowing. The ventral thenar muscle can be seen to enlarge during contraction and bulge dorsally into the tunnel as it compresses and narrows the MN, which appears somewhat more pronounced on the right side.

Case \#7 is a 56-year-old female with complaints of left upper extremity numbness and tingling for 4 months, who presented with a positive Phalen test and positive Tinel test at the carpal canal. The left median DML was $3.8 \mathrm{~ms}$ (ulnar $2.7 \mathrm{~ms}$ ) and the median D-1 DSL was $3.3 \mathrm{~ms}$ (radial $2.6 \mathrm{~ms}$ ). NMUS of the left wrist revealed increased CSA of the MN $\left(16 \mathrm{~mm}^{2}\right)$. This data was consistent with mild CTS.

Longitudinal imaging of the MN (Fig. 12) reveals a pre-stress nerve diameter of $0.21 \mathrm{~cm}$ that decreased to $0.14 \mathrm{~cm}$ during stress, which represents a $33 \%$ compressive narrowing.

Case \#8 is a 71-year-old male with complaints of right upper extremity pain, numbness, tingling, and weakness, for 3 months. Phalen and Tinel testing was normal. The right median DML was $4.4 \mathrm{~ms}$ (ulnar $2.8 \mathrm{~ms}$ ) and the median D-1 DSL was $3.3 \mathrm{~ms}$ (radial $2.6 \mathrm{~ms}$ ). NMUS of the right wrist revealed increased CSA of the $\mathrm{MN}\left(12 \mathrm{~mm}^{2}\right)$. This data was consistent with mild CTS.

Longitudinal imaging of the MN (Fig. 13) reveals a pre-stress nerve diameter of $0.22 \mathrm{~cm}$ that decreased to $0.14 \mathrm{~cm}$ during stress, which represents a $36 \%$ compressive narrowing.

Case \#9 is a 75-year-old female with complaints of right upper extremity pain, numbness, and tingling for 1 year, who presented with a positive Phalen test and positive Tinel test at the carpal canal. The right median DML was $3.9 \mathrm{~ms}$ (ulnar $2.7 \mathrm{~ms}$ ) and the median D-1 DSL was $2.9 \mathrm{~ms}$ (radial $2.5 \mathrm{~ms}$ ), median D-4 DSL was $3.7 \mathrm{~ms}$ (ulnar $3.0 \mathrm{~ms}$ ) and the median palmar mixed nerve latency was $2.2 \mathrm{~ms}$ (ulnar $1.8 \mathrm{~ms}$ ), for a combined sensory index of $1.5 \mathrm{~ms}$ (normal maximum $0.9 \mathrm{~ms}$ ). NMUS of the right wrist revealed increased CSA of the MN $\left(12 \mathrm{~mm}^{2}\right)$.

This data was consistent with mild CTS.

Longitudinal imaging of the MN (Fig. 14) reveals a prestress nerve diameter of $0.12 \mathrm{~cm}$ that decreased to $0.07 \mathrm{~cm}$ during stress, which represents a $42 \%$ compressive narrowing

Case \#10 is a 48-year-old female with complaints of right upper extremity pain, numbness, and tingling, for 
Fig. 11 CTS patient \#6. Longitudinal view of the median nerves pre-stress (a, b) and during stress $(\mathbf{c}, \mathbf{d})$. The median nerve is the darker, hypoechoic linear (or tubular) structure bordered by a thin hyperechoic rim (epineurium) traversing from the left to the right side of the image. The brighter, hyperechoic tubular structure with a fibrillar pattern just below the nerve is the flexor tendon. Note that during stress there is compressive narrowing, seen as a depression in the median nerve at the "A" markers (centrally and slightly to the right), measured as both nerve diameters decrease to $0.05 \mathrm{~cm}$, from a pre-stress diameter of $0.11 \mathrm{~cm}$ left and $0.09 \mathrm{~cm}$ right. It appears that during contraction the thenar muscle mass located ventrally (above the nerve in this image) is bulging dorsally into the tunnel against the median nerve (especially for the right median nerve $(\mathbf{d}))$ (a) CTS Patient \#6 Pre-Stress Left Image:

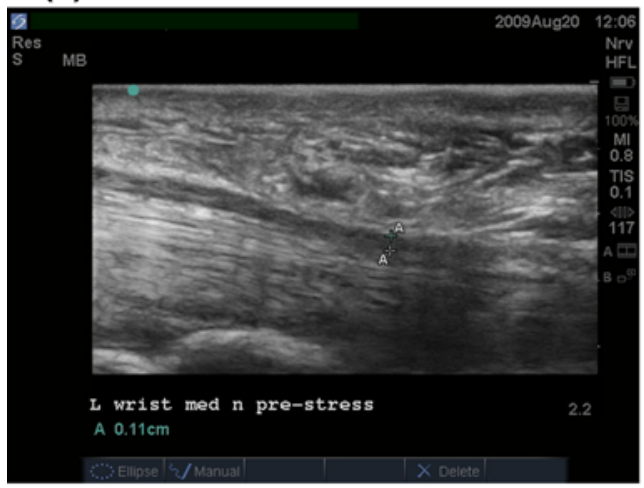

(b) CTS Patient \#6 Pre-Stress Right Image:

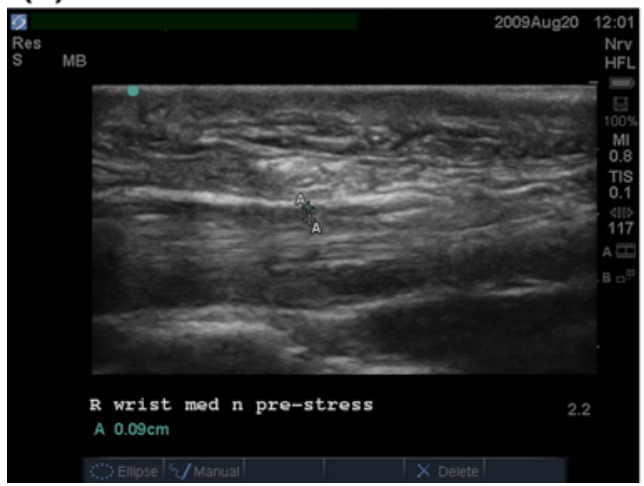

(c)CTS Patient \#6 Stress Left Image:

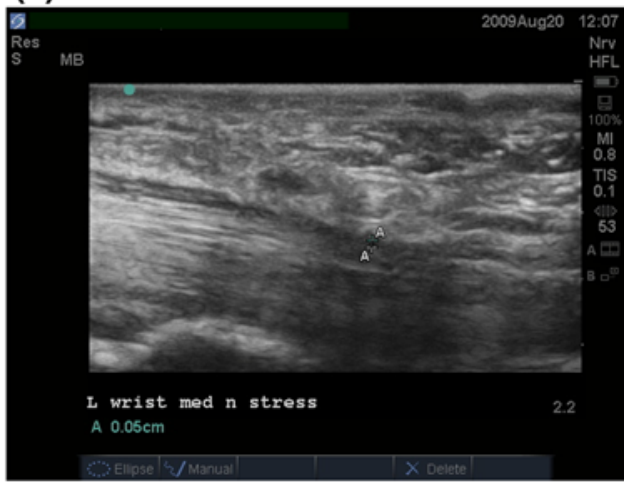

(d)CTS Patient \#6 Stress Right Image:

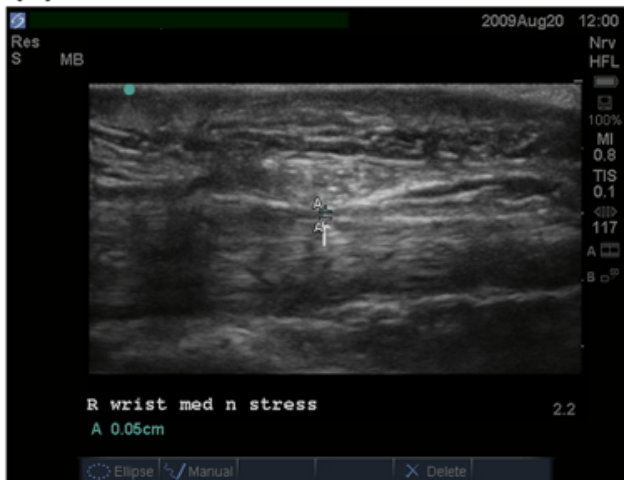

1 month, who presented with a positive Tinel test at the carpal canal (Phalen negative). The right median DML was $5.3 \mathrm{~ms}$ (ulnar $2.6 \mathrm{~ms}$ ) and the median D-1 DSL was $4.5 \mathrm{~ms}$ (radial $2.4 \mathrm{~ms}$ ). Needle electromyography of the right upper limb was entirely normal except for increased insertional activity and rare fibrillations in the thenar muscle (opponens pollicis). NMUS of the right wrist revealed increased CSA of the $\mathrm{MN}\left(17 \mathrm{~mm}^{2}\right)$. This data was consistent with moderate CTS.

Longitudinal imaging of the MN (Fig. 15a, b) reveals a pre-stress nerve diameter of $0.23 \mathrm{~cm}$ that decreased to $0.09 \mathrm{~cm}$ during stress, which represents a $61 \%$ compressive narrowing.

Transverse images were also obtained (Fig. 15c, d) and demonstrated a pre-stress nerve diameter of $0.22 \mathrm{~cm}$ that decreased to $0.14 \mathrm{~cm}$, which represents a $36 \%$ compressive narrowing. These transverse images were obtained at the distal carpal canal with the wrist in extension, which offers an "open mouth" type of view as if looking "down the throat" of the tunnel (Fig. 16).

\section{Discussion}

Carpal tunnel imaging with NMUS has been used to demonstrate enlargement of the $\mathrm{MN}$ at the level of the pisiform and is well described as a pathologic finding in CTS $[4,5]$. Other changes, such as flattening or "notching" of the MN in the distal CT have been observed, but not as consistently as nerve enlargement in the proximal carpal canal [4, 5]. Some prior studies have used ultrasound imaging to identify a decrease in nerve "sliding" within the CT during passive index finger motion [7]. Nakamichi and Tachibana [7] observed during motion that the MN slides transversely to a "freer" position in the CT with less pressure in normal individuals, but in CTS there is restricted MN motion (decreased sliding) and more exposure to compression. Another study used active contraction with fingertip loading to demonstrate increased pressure within the CT during index finger pinch grip [8]. However, none of these studies challenged or imaged the MN directly within the CT during active muscle contraction and tendon tightening to observe dynamic compression of the nerve in CTS patients.

The performance of prehensile hand movement requires that the primary movers of the thumb (abductor pollicis brevis and opponens pollicis) are fixated at their base of attachment where they "anchor" to the transverse carpal ligament (TCL) $[9,10]$. Fixation at the base allows muscle contraction to pull the thumb towards the other digits to generate maneuvers for controlled grasping. It is the solid immobile base of attachment that prepares the muscles to 
(a) CTS Patient \#7 Pre-Stress Image:

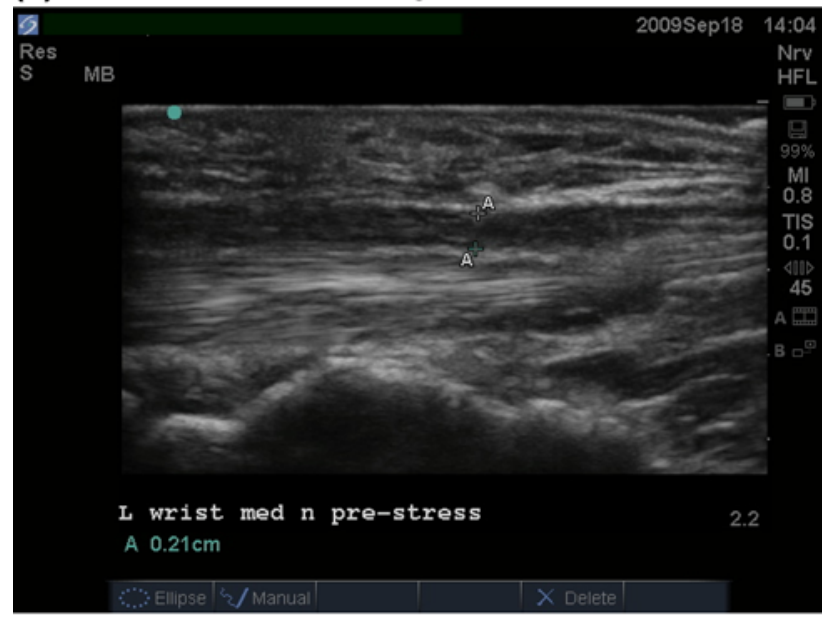

(b) CTS Patient \#7 Stress Image:

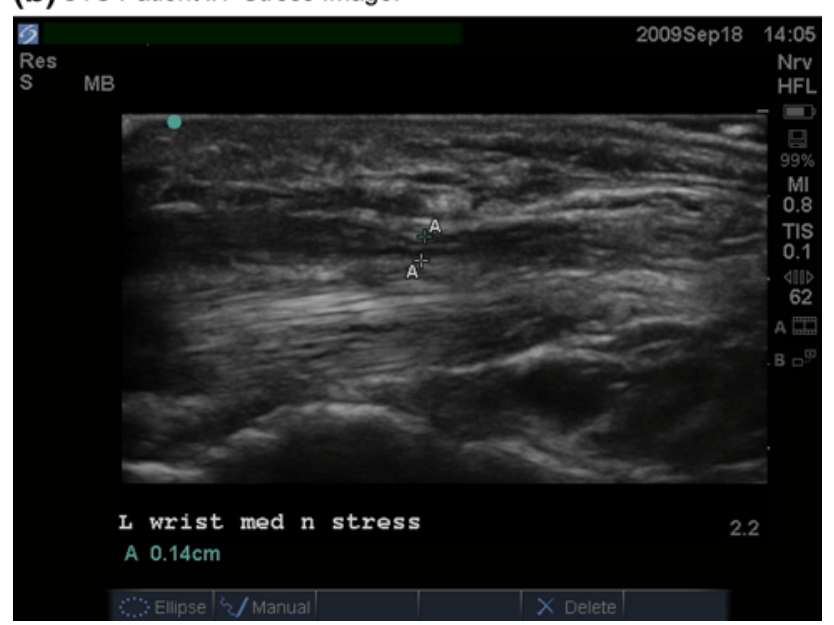

Fig. 12 CTS patient \#7. Longitudinal view of the median nerve prestress (a) and during stress (b). The median nerve is the darker, hypoechoic linear (or tubular) structure bordered by a thin hyperechoic rim (epineurium) traversing from the left to the right side of the image. The brighter, hyperechoic tubular structure with a fibrillar pattern just below the nerve is the flexor tendon. Note that during stress (b) there is compressive narrowing, seen as a depression or notching of the median nerve at the " $\mathrm{A}$ " markers (centrally and slightly to the right), measured as a diameter decrease to $0.14 \mathrm{~cm}$, from a pre-stress diameter of $0.21 \mathrm{~cm}$. It appears that during contraction the thenar muscle mass located ventrally (above the nerve in this image) is bulging dorsally into the tunnel against the median nerve, and a portion of the flexor tendon has tightened and is bulging upward (ventrally) into the nerve, effectively "sandwiching" the nerve between the muscle and tendon

freely move the thumb about as required. However, during sustained grasp or pinch, such as squeezing a hard ball, the thumb tip becomes immobile and the anchor becomes the mobile segment. This action simulates firmly holding a pencil, the most common form of prehensile hand activity [9]. During this action, thenar contraction pulls the TCL taut (instead of moving the thumb), and the muscle bulges dorsally into the CT. Simultaneously, the flexor tendons to (a) CTS Patient \#8 Pre-Stress Image:

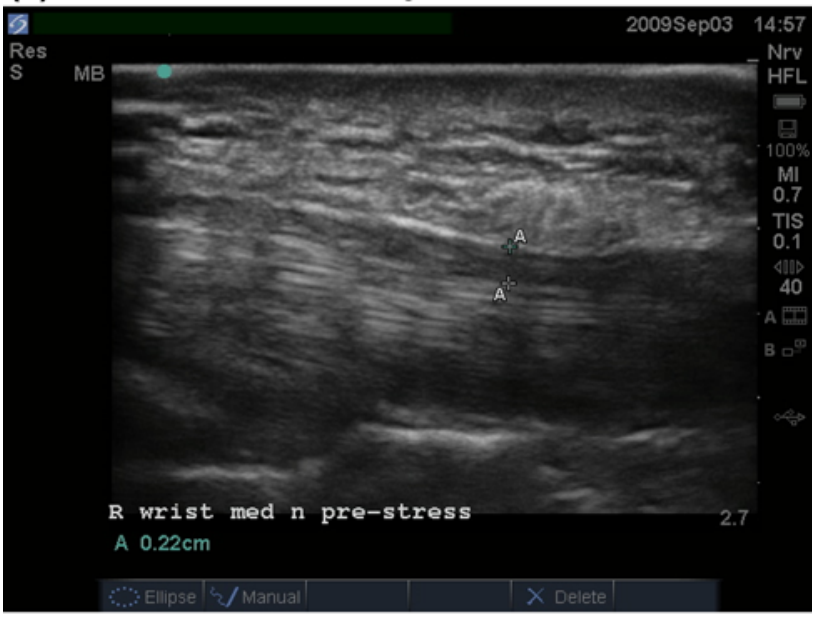

(b) CTS Patient \#8 Stress Image:

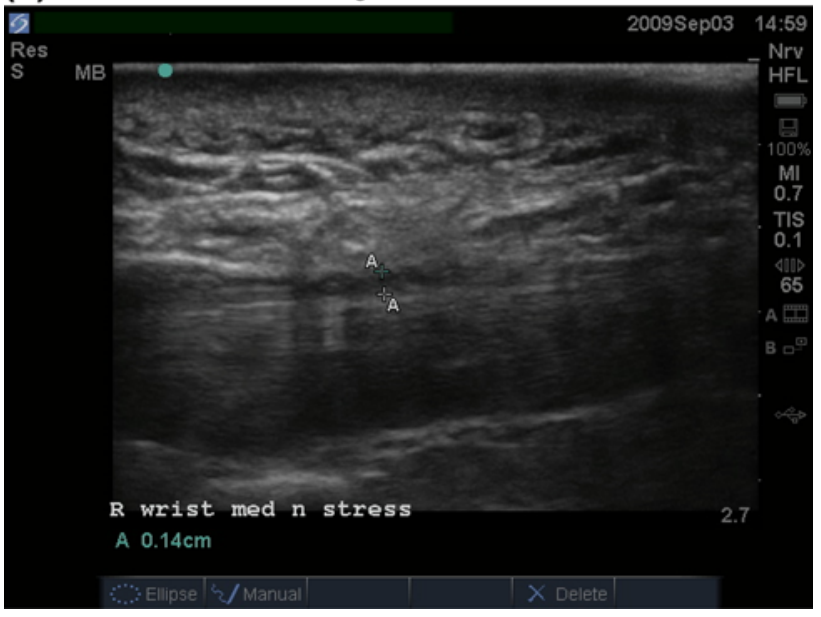

Fig. 13 CTS patient \#8. Longitudinal view of the median nerve prestress (a) and during stress (b). The median nerve is the darker, hypoechoic linear (or tubular) structure bordered by a thin hyperechoic rim (epineurium) traversing from the left to the right side of the image. The brighter, hyperechoic tubular structure with a fibrillar pattern just below the nerve is the flexor tendon. Note that during stress (b) there is compressive narrowing, seen as a depression along the median nerve near the "A" markers (centrally and slightly to the right), measured as a diameter decrease to $0.14 \mathrm{~cm}$, from a pre-stress diameter of $0.22 \mathrm{~cm}$. It appears that during contraction the thenar muscle mass located ventrally (above the nerve in this image) is bulging dorsally into the tunnel and against the median nerve

the thumb and digits are pulled taut towards the underside of the TCL, which compresses the juxtaposed MN. The ultrasound images demonstrate notching, flattening, or compression of the nerve during dynamic stress testing maneuvers (see Figs. 6, 7, 8, 9, 10, 11, 12, 13, 14, 15). It appears that the roof (TCL) of the tunnel tightens and lowers at the same time the floor (flexor tendons) of the tunnel tightens and raises, thereby compressing the nerve.

Dynamic studies with digital video recording by the author in several CTS patients have demonstrated that the median nerve is actively compressed during pinch activity. 
(a) CTS Patient \#9 Pre-Stress Image:

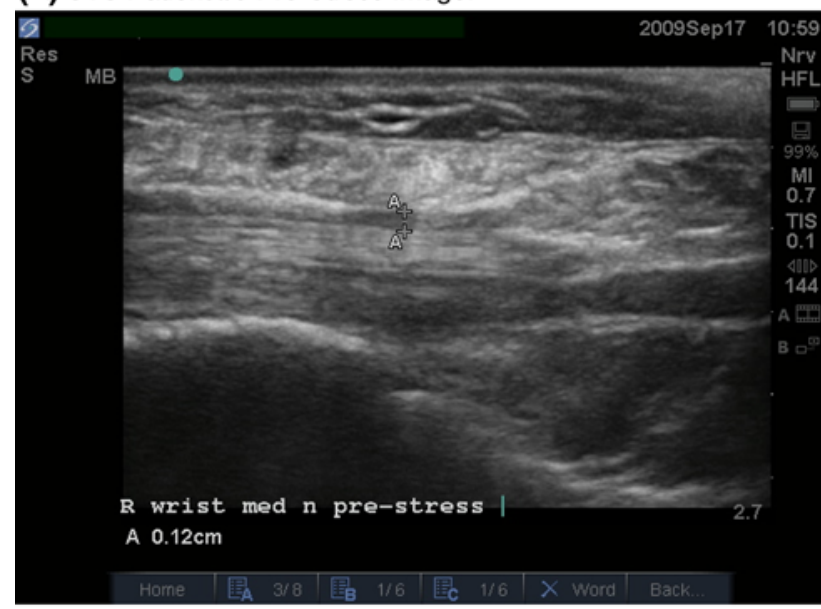

(b) CTS Patient \#9 Stress Image:

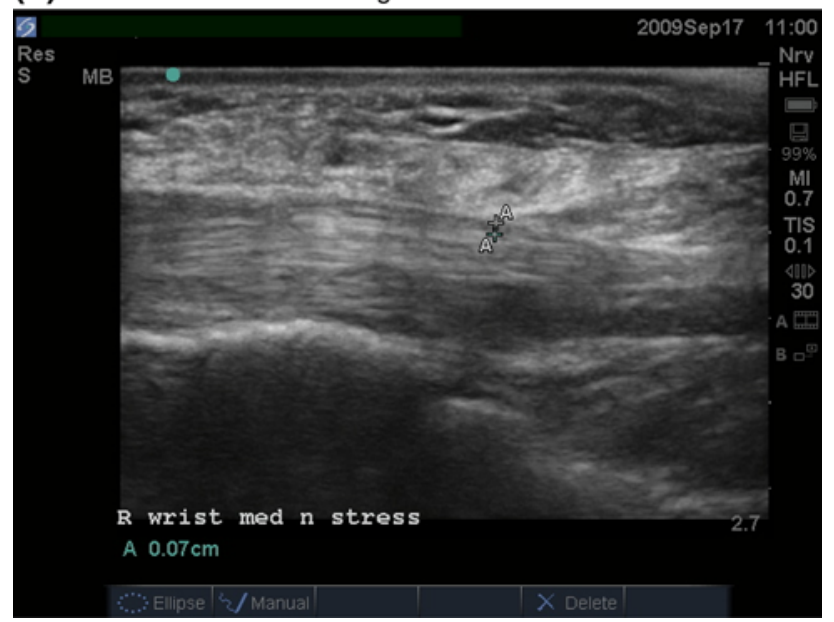

Fig. 14 CTS patient \#9. Longitudinal view of the median nerve prestress (a) and during stress (b). The median nerve is the darker, hypoechoic linear (or tubular) structure bordered by a thin hyperechoic rim (epineurium) traversing from the left to the right side of the image. The brighter, hyperechoic tubular structure with a fibrillar pattern just below the nerve is the flexor tendon. Note that during stress (b) there is compressive narrowing, seen as a depression or flattening of the median nerve at the " $\mathrm{A}$ " markers (centrally and slightly to the right), measured as a diameter decrease to $0.07 \mathrm{~cm}$, from a pre-stress diameter of $0.12 \mathrm{~cm}$. It appears that during contraction the thenar muscle mass located ventrally (above the nerve in this image) is bulging dorsally into the tunnel and against the median nerve

This is particularly obvious with transverse imaging in the distal tunnel region (see Fig. 15c, d and 16a, b), but can be challenging to maintain the image as the thenar muscle contracts and "pushes" the ultrasound transducer off the nerve. It is interesting to note in patients or individuals without CTS that the nerve simply "slides" out of the way, avoiding compression. This observation supports the theories of Hunter [11] and Phalen [12] regarding fibrous fixation of the median nerve in CTS. It may be that fibrosis within the carpal canal of CTS patients prevents the MN from sliding out of harms way during routine hand activity. In fact, Sucher suggested that manipulation and stretching may be successful in alleviating symptoms of CTS by breaking up the adhesions or fibrous fixations [10].

It may also be noteworthy that the MN compression observed in this study was always located in the mid-distal carpal canal. This observation is consistent and supportive of prior observations by Kimura [13] that maximal nerve conduction slowing in CTS (and even normal subjects) occurs $2-4 \mathrm{~cm}$ distal to the origin of the TCL, which is effectively the mid-distal carpal tunnel. Kimura's observations were subsequently verified by work from Seror [14], who also used a segmental ("inching") nerve conduction technique.

The implications for understanding the pathomechanics that contribute to development of CTS can be applied to treatment, and perhaps prevention of MN injury. It is not clearly understood why repetitive or vigorous hand activity leads to development of CTS in some individuals while others are spared. Researchers have observed contractile cells in the TCLs of CTS patients and suggested that they were undergoing a constant state of contraction [15]. Sucher [10] observed and quantified mechanical restriction at the wrist in CTS patients, which could correlate with tightness in the TCL. Subsequent work by Sucher and Hinrichs [16] identified that the distal band of the TCL is the most significant, because it is thicker, and thus correlates with this region being the site of maximal nerve conduction slowing and maximal nerve compression seen on NMUS. It is unknown whether such patients had preexisting abnormalities that allowed CTS to develop, or if specific patterns of hand movement created the abnormalities that subsequently led to MN injury. However, increased tightness can develop within the TCL due to ongoing repetitive or sustained contractions of the thenar muscles, possibly combined with contracting myofibroblasts in the TCL, all of which leads to further foreshortening and pressure on the MN. Finally, there is apparent hypertrophy of the thenar muscle mass due to perpetual contraction, which causes these muscles to protrude into the carpal tunnel during activity.

The pathologic mechanics observed in the CT during activity suggest a multi-factorial cause of CTS: increased intracarpal pressure [2,7], decreased nerve mobility (from fibrous fixation) [7, 12], nerve deformation (compression, stretching, traction) [11], increased stiffness of the synovium and flexor retinaculum (TCL) [7], relative thenar hypertrophy or increased muscle mass with intrusion into the $\mathrm{CT}$, and tendon thickening/tightening during activity. It is these latter two processes that appear to contribute most to compression observed with NMUS, by tightening and lowering the TCL at the same time the floor is tightened and raised during prehensile activity (thenar flexion and 
(a) cTS Patient \#10 Pre-Stress Image:

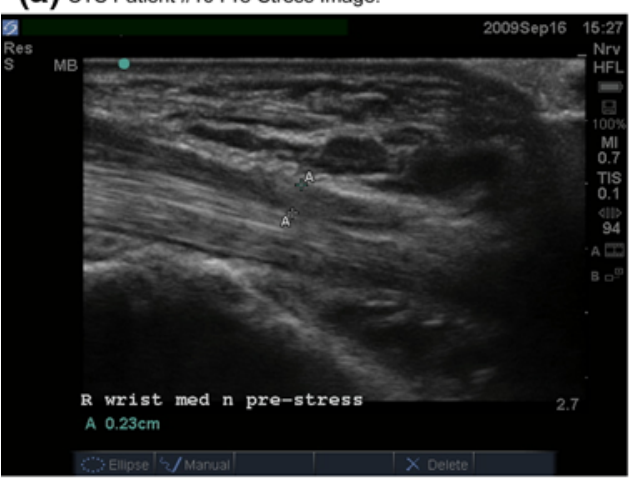

(b) CTS Patient \#10 Stress Image:

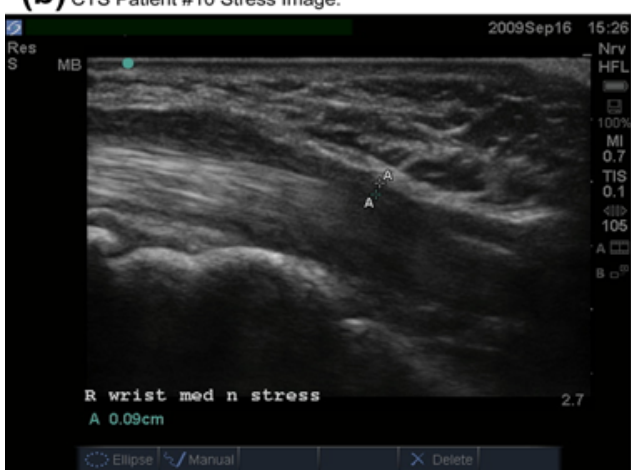

Fig. 15 CTS Patient \#10. Longitudinal view of the median nerve prestress (a) and during stress (b). The median nerve is the darker, hypoechoic linear (or tubular) structure bordered by a thin hyperechoic rim (epineurium) traversing from the left to the right side of the image. The brighter, hyperechoic tubular structure with a fibrillar pattern just below the nerve is the flexor tendon. Note that during stress (b) there is compressive narrowing, seen as a depression or notching of the median nerve near the "A" markers (centrally and slightly to the right), measured as a diameter decrease to $0.09 \mathrm{~cm}$, from a pre-stress diameter of $0.23 \mathrm{~cm}$. It appears that during contraction the thenar muscle mass located ventrally (above the nerve in this image) is bulging dorsally into the tunnel against the median nerve, and a portion of the flexor tendon has tightened and is bulging upward (ventrally) into the nerve, effectively "sandwiching" the nerve between the muscle and tendon. Transverse scans (c, d) illustrate the large hypoechoic (darker) wedge-shaped thenar muscle

opposition to the first two fingers). This obvious compressive action could support a new theory that most CTS is not idiopathic, in contrast to prior claims [1, p. 67, 106] Rather, the development of CTS may simply be a selfcorrective mechanism, where overuse hand activity causes muscle and tendon behavior to compress the nerve supplying the muscle that generates the activity, thereby leading to weakness and atrophy of that muscle, which allows the compression to ease enough to allow the nerve to recover.

Studies [10, 16-19] have demonstrated osteopathic manipulative treatment (OMT) of the wrist can increase the size of the CT, alleviate CTS symptoms, and lead to (c) CTS Patient \#10 Pre-Stress Transverse Image:

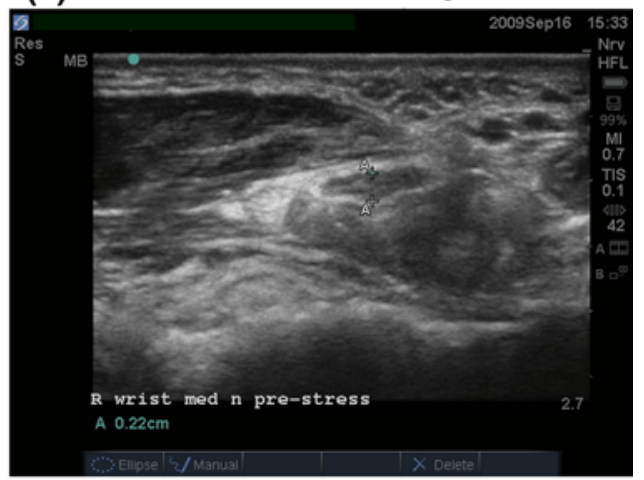

(d) CTS Patient \#10 Stress Transverse Image:

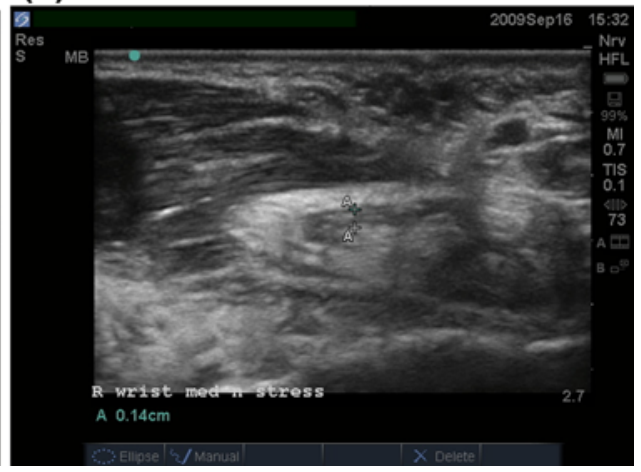

mass on the left. The median nerve is the hypoechoic ellipsoid or oval structure near the center of the image, and the " $\mathrm{A}$ " markers measure the anterior-posterior diameter of the nerve as $0.22 \mathrm{~cm}$ pre-stress (c) and $0.14 \mathrm{~cm}$ during compressive stress (d). The thenar mass can be seen to thicken and shorten as it contracts and pulls the ligamentous (TCL-transverse carpal ligament) attachment to the left (lateral) and flattens a portion of the ligament that lies directly above the nerve (seen as a hyperechoic or whiter band directly above the most ventral "A" marker on the nerve), causing the nerve to elongate transversely as it is flattened and compressed downward (dorsally). Simultaneously, the contracting flexor muscles tightened the flexor tendons ventrally, and these can be seen as hyperechoic oval or round areas (d) directly below (dorsally) the median nerve, just beneath the lower "A" marker, adding to the compressive effect by creating a more rigid and unyielding tunnel floor

improved nerve conduction values. The most essential technique used for OMT of CTS involves thenar abduction and extension, which applies traction to the TCL, most likely elongating the TCL and increasing space within the CT. Another effect of the OMT that was not previously considered probably involves elongation of the thenar muscles and release of the focal prominence that intrudes into the tunnel during active contraction. An additional essential component of treatment involves wrist and digit hyperextension to stretch and elongate the flexor tendons. The present study of cases with NMUS imaging suggests that the mechanism of MN release with OMT impacts several of the suspected factors causing CTS. Stretching 
Table 2 CTS patients' measurement data

\begin{tabular}{|c|c|c|c|c|c|c|c|c|}
\hline CASE \# & $\mathrm{H} \& \mathrm{P}$ & NCS & EMG & $\begin{array}{l}\mathrm{CSA} \\
\left(\mathrm{mm}^{2}\right)\end{array}$ & WFR & $\begin{array}{l}\text { Pre-stress } \\
\text { diameter }(\mathrm{cm})\end{array}$ & $\begin{array}{l}\text { Stress } \\
\text { diameter }(\mathrm{cm})\end{array}$ & $\%$ Change \\
\hline 1 & + & + & $\mathrm{n} / \mathrm{a}$ & 11 & 2.75 & 0.16 & 0.11 & -31.25 \\
\hline 2 & + & + & $\mathrm{n} / \mathrm{a}$ & 11 & $\mathrm{n} / \mathrm{a}$ & 0.20 & 0.12 & -40.0 \\
\hline 3 & + & + & $\mathrm{n} / \mathrm{a}$ & 10 & 2.5 & 0.20 & 0.13 & -35.0 \\
\hline 4 & + & + & $\mathrm{n} / \mathrm{a}$ & 10 & 2.0 & 0.20 & 0.13 & -35.0 \\
\hline 5 & + & + & $\mathrm{n} / \mathrm{a}$ & 13 & $\mathrm{n} / \mathrm{a}$ & 0.18 & 0.14 & -22.2 \\
\hline $6(\mathrm{Rt})$ & + & + & $\mathrm{n} / \mathrm{a}$ & 13 & $\mathrm{n} / \mathrm{a}$ & 0.11 & 0.05 & -54.5 \\
\hline$(\mathrm{Lt})$ & + & + & $\mathrm{n} / \mathrm{a}$ & 13 & $\mathrm{n} / \mathrm{a}$ & 0.09 & 0.05 & -44.4 \\
\hline 7 & + & + & $\mathrm{n} / \mathrm{a}$ & 16 & $\mathrm{n} / \mathrm{a}$ & 0.21 & 0.14 & -33.3 \\
\hline 8 & + & + & $\mathrm{n} / \mathrm{a}$ & 12 & $\mathrm{n} / \mathrm{a}$ & 0.22 & 0.14 & -36.4 \\
\hline 9 & + & + & $\mathrm{n} / \mathrm{a}$ & 12 & $\mathrm{n} / \mathrm{a}$ & 0.12 & 0.07 & -41.7 \\
\hline 10 & + & + & + & 17 & $\mathrm{n} / \mathrm{a}$ & 0.23 & 0.09 & -60.9 \\
\hline Mean change (diameter) & & & & & & & & -39.51 \\
\hline
\end{tabular}

$H \& P$ history and physical, NCS nerve conduction study, EMG needle electromyogram, CSA median nerve cross-sectional area, WFR ratio of median nerve CSA at wrist divided by CSA in distal forearm (normal < 1.5), "+" signifies positive or abnormal and consistent with CTS, "_" signifies negative or normal, $n / a$ not applicable or not obtained

(a) Ultrasound technique for distal tunnel transverse pre-stress imaging

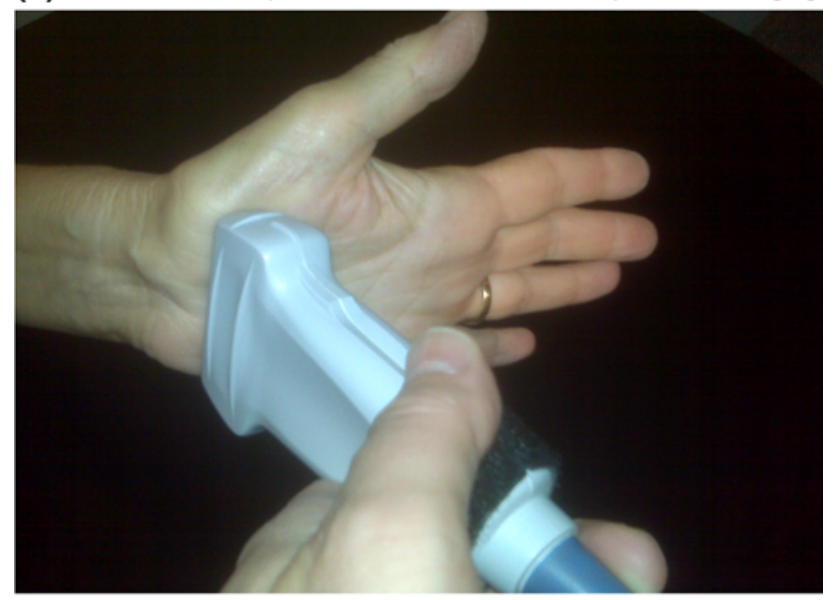

(b) Ultrasound technique for distal tunnel transverse stress imaging:

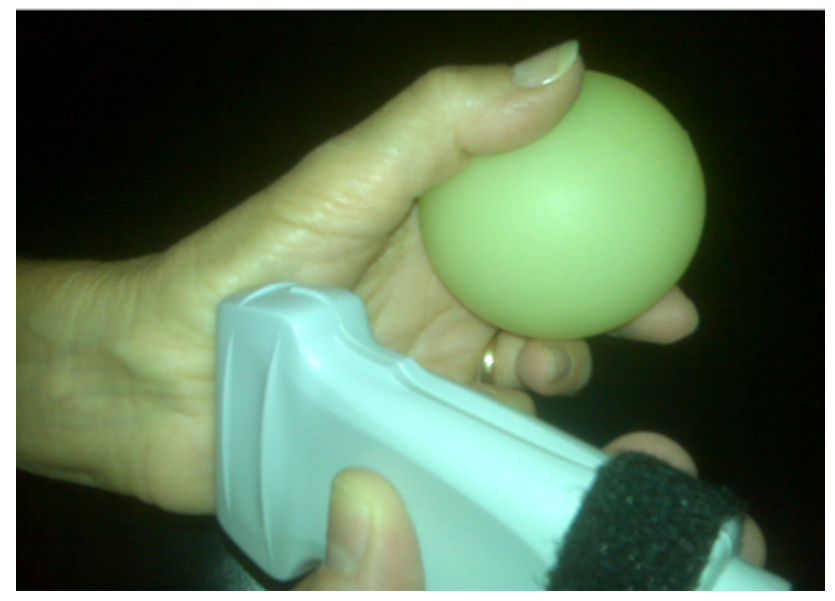

Fig. 16 Transverse scan technique demonstrates method of obtaining a distal tunnel "open-mouth" view in the pre-stress (a) and stress (b) positions the TCL elongates that structure and leads to increased space within the CT, decreasing pressure on the MN. Simultaneously, alleviating thenar muscle tightness will lead to less protrusion into the CT. Elongating the flexor tendons will decrease thickening and tightening on the dorsal side (floor) of the tunnel and thereby alleviate some pressure from the MN. Each of these maneuvers, alone or in combination, will prepare the normal CT for enhanced activity tolerance, and could become a valuable component of a program for prevention of CTS.

\section{Conclusion}

NMUS imaging of the CT provides a new "window" through which clinicians can directly observe and better understand the pathomechanics involved in the development of CTS. Imaging of MN compression during prehensile hand activity, previously suspected as a contributory cause of nerve injury, can be documented and measured. In addition, thenar muscle prominence and protrusion into the CT has been identified as a potential etiologic factor that was heretofore unsuspected. These new understandings of the mechanisms related to the cause of CTS adds confirmation to prior work [10, 16-19] that advocates aggressive OMT and stretching approaches to management. Application of NMUS with stress testing (DST) potentially opens a door to prevention of CTS.

Open Access This article is distributed under the terms of the Creative Commons Attribution Noncommercial License which permits any noncommercial use, distribution, and reproduction in any medium, provided the original author(s) and source are credited. 


\section{References}

1. Rosenbaum RB, Ochoa JL. Carpal tunnel syndrome and other disorders of the median nerve. 2nd ed. Amsterdam: Butterworth Heinemann; 2002.

2. Rojviroj S, Sirichativapee W, Kowsuwon W, Wongwiwattananon $\mathrm{J}$, et al. Pressures in the carpal tunnel. A comparison between patients with carpal tunnel syndrome and normal subjects. J Bone Joint Surg Br. 1990;72-B:516-8.

3. Robinson LR, Micklesen PJ, Wang L. Optimizing the number of tests for carpal tunnel syndrome. Muscle Nerve. 2000;23:1880-2.

4. Beekman R, Visser LH. Sonography in the diagnosis of carpal tunnel syndrome: a critical review of the literature. Muscle Nerve. 2003;27:26-33.

5. Bodner G. Nerve compression syndromes. In: Peer S, Bodner G, editors. High-resolution sonography of the peripheral nervous system. 2nd Rev ed. Berlin: Springer-Verlag; 2008.

6. Hobson-Webb LD, Padua L. Median nerve ultrasonography in carpal tunnel syndrome: findings from two laboratories. Muscle Nerve. 2009;40:94-7.

7. Nakamichi K, Tachibana S. Restricted motion of the median nerve in carpal tunnel syndrome. J Hand Surg Br. 1995;20B:460 4.

8. Keir PJ, Bach JM, Rempel DM. Fingertip loading and carpal tunnel pressure: differences between a pinching and a pressing task. J Orthop Res. 1998;16:112-5.

9. Kapandji IA. The physiology of the joints, vol 1: upper limb. Edinburgh: Churchill Livingstone; 1979.
10. Sucher BM. Palpatory diagnosis and manipulative management of carpal tunnel syndrome. J Am Osteopath Assoc. 1994;4:647-63.

11. Hunter J. Recurrent carpal tunnel syndrome, epineural fibrous fixation, and traction neuropathy. Hand Clin. 1991;7(3):491-504.

12. Phalen GS. Reflections on 21 years' experience with the carpal tunnel syndrome. JAMA. 1970;212:1365-7.

13. Kimura J. Mononeuropathies and entrapment syndromes. In: Kimura J, editor. Electrodiagnosis in diseases of nerve and muscle. Philadelphia: FA Davis; 1983.

14. Seror P. Orthodromic inching test in mild carpal tunnel syndrome. Muscle Nerve. 1998;21:1206-8.

15. Allampallan K, Chakraborty J, Bose KK, Robinson J. Explant culture, immunofluorescence and electron-microscopic study of flexor retinaculum in carpal tunnel syndrome. J Occup Environ Med. 1996;38:264-71.

16. Sucher BM, Hinrichs RN. Manipulative treatment of carpal tunnel syndrome: biomechanical and osteopathic intervention to increase the length of the transverse carpal ligament. J Am Osteopath Assoc. 1998;98:679-86.

17. Sucher BM. Myofascial release of carpal tunnel syndrome. J Am Osteopath Assoc. 1993;93:92-101.

18. Sucher BM. Myofascial manipulative release of carpal tunnel syndrome: documentation with MRI. J Am Osteopath Assoc. 1993;93:1273-8.

19. Sucher BM. Palpatory diagnosis and manipulative management of carpal tunnel syndrome: Part 2. "Double crush" and thoracic outlet syndrome. J Am Osteopath Assoc. 1995;95:471-9. 